\title{
Instructional effects on semantic satiation
}

\author{
STEPHEN A. MADIGAN AND ALLAN PAIVIO
}

UNIVERSITY OF WESTERN ONTARIO

Relatively small satiation effects were found in a study of the effects of instructional variables on semantic satiation. The largest satiation effects were produced when Ss were instructed to consider word meanings during repetition of words, and in addition rated words on semantic differential scales on which the mid-point was defined as indicating "meaninglessness" as well as "neutrality of irrelevancy." It was concluded that semantic satiation effects may be difficult to obtain without the use of such instructions when the semantic differential is employed to measure meaning loss following verbal repetition.

Yelen \& Schulz (1963), and Schulz, Weaver, \& Radtke (1965) have reported failures to obtain the semantic satiation effect described by Lambert \& Jakobovits (1960), variously finding little or no meaning loss (satiation), or increased intensity of meaning (generation). These investigators have generally employed procedures closely resembling those of Lambert and Jakobovits, although the scoring method used by Yelen and Schulz differed from that of Lambert and Jakobovits. However, the studies may have varied in at least one aspect of the procedure. Lambert and Jakobovits apparently gave their Ss instructions on the use of the semantic differential (S.D.) in which the midpoint of S.D. scales was defined as indicating judgments of meaninglessness as well as the neutrality or irrelevancy indicated by the standard instructions (Osgood, Suci, \& Tannenbaum, 1957, p. 83) $0^{1}$ Lambert \& Jakobovits (1960) specified such a definition of a scale midpoint in the introduction to their report, but did not explicitly describe the instructions that were given to Ss concerning the use of the scales. It is possible, therefore, that other investigators have been unaware of the particular procedure used and employed standard instructions only. The present study tested the effects of the two types of instructions on S.D. ratings of words following verbal repetition. It was hypothesized that the definition of a scale midpoint as indicating "meaninglessness" would produce greater satiation effects than would the standard instructions inasmuch as the "meaninglessness" label could serve as a cue to $\mathrm{Ss}$ to report meaning loss. A second instructional variable, simultaneously investigated, was the Ss' general orientation to the repetition and rating tasks. A previous unpublished study had indicated that an orientation to consider word meanings during repetition resulted in more generation of meaning than did a "task" orientation, under which Ss were instructed to attend carefully to the experimental tasks. In view of the reported sensitivity of satiation effects, it was felt that the orientation variable would promote different amounts and directions of rating change.

\section{Method}

Ss were 88 introductory psychology students, 25 male and 63 female. The design was a 2 by 2 factorial (Definition by Orientation). Definition was varied by defining the midpoint of S.D. scales in one condition as indicating "meaninglessness, neutrality, or irrelevancy," and in the other condition as indicating "neutrality or irrelevancy" only. Instructions to Ss either to attend to word meanings or to the repetition task comprised the meaning and task orientations, respectively. The procedure employed was similar to the Lambert \& Jakobovits (1960) method for their experimental group. All Ss initially rated five words (family, me, truth, death, child) on each of six S.D. scales (good-bad, kind-cruel, emotional-rational, violentgentle, active-passive, strong-weak). This was followed by 30 periods (five words by six scales) of $15 \mathrm{sec}$. repetition of a word and re-rating of the word on a scale. The procedure differed from Lambert and Jakobovits in that Ss were isolated in cubicles and heard tape-recorded instructions and repetition words via headphones. There were four versions of the basic instructions, each relating to one of the four experiment conditions: meaning orientation with "meaninglessness" instructions vis-a-vis the scale midpoint, meaning orientation without the "meaninglessness" set, and task orientation with or without the "meaninglessness" instructions. Ss were instructed to repeat words aloud along with the recorded repetitions. The same set of word repetitions was presented to all Ss to insure some standardization of the volume and rate of Ss' repetitions.

\section{Results and Discussion}

A polarity-difference score (P.D.S.), i.e., the difference between the sum of post-repetition polarities and the sum of pre-repetition polarities for the 30 word-scale units, was computed for each S. Negative scores thus indicated decreased polarity (satiation), and positive scores indicated increased polarity (generation). A 2 by 2 analysis of variance showed a significant interaction ( $F=5.51, \mathrm{df}=1 / 84, \mathrm{p}<.05)$ but no main effects. Figure 1 shows that, with a meaning orientation, satiation occurred with the "meaninglessness" definition of the midpoint, and generation without such a condition. This pattern was reversed for the task orientation. However, the mean amount of rating change associated with any of the treatment conditions was relatively small. The mean P.D.S. per word (averaging the P.D. scores in Fig. 1 by the number of words used) for each treatment group was: meaning orientation with "meaninglessness" set, -.24 ; meaning orientation without set, .75; task orientation with set, .26; and task orientation without 


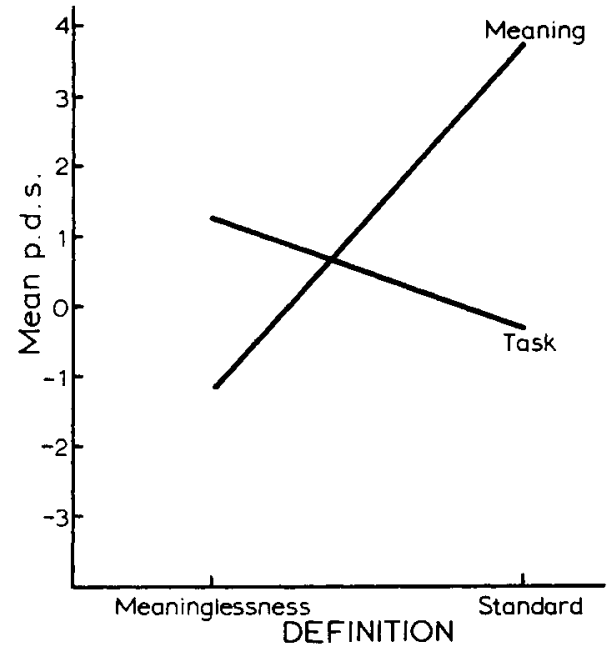

Fig. 1. Definition by orientation interaction for polarity-difference scores.

set, -.07. In support of the hypothesis, the P.D.S. of -.24 for the condition with the meaning orientation and the "meaninglessness" instructions was the greatest amount of satiation found in the study, but a reduced polarity of -.24 scale units per word over six scales represents at best a very weak "meaning loss." While the magnitude of a P.D.S. should be evaluated in relation to the initial degree of polarization of ratings, the fact that fewer than one-quarter of the total number of post-repetition ratings showed decreased polarization over initial ratings reinforces the conclusion that the observed satiation effects were small.

Three additional analyses were done. First, the absolute amounts of rating change (i.e., P.D. scores without signs) were analysed. There was a significant main effect of orientation $(F=6.69, \mathrm{df}=1 / 84, \mathrm{p}<.05)$, with the meaning orientation producing more changes per se than the task orientation. The effect of instructions to "carefully consider word meanings" during repetition apparently encouraged $S s$ to change their ratings. Second, the relative tendencies towards satiation and generation within Ss was ascertained by tallying the frequencies of rating changes producing increased and decreased polarity. Each $S$ was thus given a generation and a satiation score. An analysis of generation scores showed that they were equally distributed over the treatment groups. An analysis of satiation scores, however, revealed an interaction similar to that shown in Fig. $1(F=4.54, \mathrm{df}=1 / 84$, $\mathrm{p}<.05)$. Since the P.D. score for a $S$ consists essentially of the difference between his separate satiation and generation scores, and since mean generation scores did not differ between groups, the interaction of the orientation and definition variables can be interpreted as reflecting differing tendencies to satiation of meaning among the treatment groups.

The results of the present experiment, like those of Schulz et al (1965), indicate that satiation effects on S.D. ratings are difficult to obtain, inasmuch as any satiation effects that were observed were quite small and generation of meaning occurred almost equally often over the experiment as a whole. The findings suggest a need for reconsideration of the semantic differential technique for measuring satiation. Since greater satiation effects were produced by the "meaninglessness" definition than by the standard definition of the midpoint, it may be that the former served mainly as a cue to Ss to report meaning loss. If this is so, the Lambert and Jakobovits S.D. studies would not be superior in their objectivity to Smith \& Raygor's (1956) experiment, in which Ss were explicitly instructed to report meaning loss. It should be noted, however, that the use of tape-recorded repetitions in the present study departed from the procedure used by Lambert and Jakobovits and our results may be open to other interpretations.

\section{Rieferences}

Lambert, W. E., \& Jakobovits, L. A. Verbal satiation and changes in the intensity of meaning. $J$. exp. Psychol., 1960, 60, 376-383.

Osgood, C. E., Suci, G. J., \& Tannenbaum, P. H. The measurement of meaning. Urbana, Illinois: University of Illinois Press, 1957.

Schulz, R. W., Weaver, G. E., \& Radtke, R. C. Verbal satiation. Psychon. Sci., 1965, 2, 43-44.

Smith, D. E. P., \& Raygor, A. L. Verbal satiation and personality. J. verbal Learn. verbal Behav., 1963, 1, 372-377.

Yelen, D. R., \& Schulz, R. W. Verbal satiation. J. verbal Learn verbal Behav., 1963, 1, 372-377.

\section{Note}

1. Personal communication from L. A. Jakobovits, 1966. 